\title{
COMPARISON OF METHODS FOR GEOMETRIC CAMERA CALIBRATION
}

\author{
Jan Hieronymus \\ Computer Vision \\ Humboldt-Universität zu Berlin \\ Unter den Linden 6, 10099 Berlin, Germany \\ jan.hieronymus@informatik.hu-berlin.de \\ http://www2.informatik.hu-berlin.de/cv
}

Commission V/5

KEY WORDS: Geometric Camera Calibration, Distortion Modelling, Accuracy Comparison, Algorithms

\begin{abstract}
:
Methods for geometric calibration of cameras in close-range photogrammetry are established and well investigated. The most common one is based on test-fields with well-known pattern, which are observed from different directions. The parameters of a distortion model are calculated using bundle-block-adjustment-algorithms. This methods works well for short focal lengths, but is essentially more problematic to use with large focal lengths. Those would require very large test-fields and surrounding space. To overcome this problem, there is another common method for calibration used in remote sensing. It employs measurements using collimator and a goniometer. A third calibration method uses diffractive optical elements (DOE) to project holograms of well known pattern. In this paper these three calibration methods are compared empirically, especially in terms of accuracy. A camera has been calibrated with those methods mentioned above. All methods provide a set of distortion correction parameters as used by the photogrammetric software Australis. The resulting parameter values are very similar for all investigated methods. The three sets of distortion parameters are crosscompared against all three calibration methods. This is achieved by inserting the gained distortion parameters as fixed input into the calibration algorithms and only adjusting the exterior orientation. The RMS (root mean square) of the remaining image coordinate residuals are taken as a measure of distortion correction quality. There are differences resulting from the different calibration methods. Nevertheless the measure is small for every comparison, which means that all three calibration methods can be used for accurate geometric calibration.
\end{abstract}

\section{KURZFASSUNG:}

In der Nahbereichphotogrammetrie gibt es bewährte und gut untersuchte Methoden zur geometrischen Kalibrierung. Die gebräuchlichste basiert darauf, bekannte Muster eines Testfeldes aus verschiedenen Richtungen zu photographieren. Die Parameter eines Verzeichnungsmodelles werden im Rahmen einer Bündel-Block-Ausgleichung berechnet. Diese Kalibriertechnik funktioniert gut für kurzbrennweitige Optiken; eine Verwendung langbrennweitiger Optiken ist hingegen umständlich, da große Testfelder und viel Platz in der Umgebung benötigt wird. In der Fernerkundung wird deshalb die Kalibrierung mit Kollimator und Goniometer bevorzugt. Eine dritte Methode basiert auf der Analyse eines holographischen Musters, das mit Hilfe eine diffraktiven optischen Elementes (DOE) erzeugt wird. In diesem Beitrag werden diese Kalibriermethoden empirisch verglichen, mit besonderem Augenmerk auf die Genauigkeit. Eine Kamera wurde mit den genannten Methoden kalibriert. Alle Methoden geben einen Satz an Verzeichnungsparametern aus, wie sie in der photogrammetrischen Software Australis verwendet werden. Die Werte der Verzeichnungsparametersätze unterscheiden sich von Methode zu Methode nicht wesentlich. Die Verzeichnungsparametersätze werden verglichen, indem sie jeweils als nicht auszugleichende Parameter in alle drei Kalibrieralgorithmen eingesetzt werden; nur die äußere Orientierung und die gemessenen Bildkoordinaten werden ausgeglichen. Der RMS (root mean square) der Bildkoordinatenresiduen wird als vergleichbares Maß verwendet. Es weist kleine Differenzen auf, die auf die unterschiedlichen Verfahren zurückzuführen ist. Das Maß ist jedoch in allen Fällen relativ klein, sodass alle Kalibriermethoden zur präzisen geometrischen Kalibrierung verwendet werden können.

\section{INTRODUCTION}

Geometric calibration of a camera is necessary for precise measurement using images. Several methods exist to measure the distortion of a camera. In this paper test-field calibration, goniometric calibration and DOE calibration will be compared and discussed. Test-field calibration in conjunction with bundle-adjustment is relatively accurate, well investigated and established. On the other hand it is hard to use under laboratory circumstances for cameras with a focal length greater than approximately $35 \mathrm{~mm}$. The reason is, that the camera will deliver unsharp images below a certain distance to the object, i.e. the test-field. Goniometric calibration and DOE calibration instead can be used with essentially greater focal lengths, but both of them require expensive calibration equipment.
The paper is organized as following. Section 1 outlines the fundamental characteristics of the investigated calibration methods. Section 2 describes the introduced calibration methods more detailed as well as the applied distortion model. Section 3 presents a numerical comparison of the investigated calibration methods. Finally the conclusion is drawn, that all calibration methods are suitable for accurate geometric calibration.

\section{GEOMETRIC CALIBRATION METHODS}

A general overview of calibration methods is given in (Clarke and Fryer, 1998). The following investigated geometric calibration methods rely on observing well known geometric features and comparing their ideal image to the distorted real image. For many cameras the ideal image can be calculated using the pin- 
hole or perspective camera model. Several other models including generic camera models exist, which can describe also nonperspective cameras (Luber and Reulke, 2010). The goal of a geometric calibration is to measure the interior orientation of a camera, which comprises the focal length and a description of the differences between the ideal image and the distorted real image. Those differences are usually represented by the parameters of a distortion model. In this paper the distortion model implemented in the photogrammetric software Australis is used.

Several calibration methods exist to acquire couples of ideal and distorted images. In this paper test-field calibration, goniometric calibration and DOE calibration are compared.

With all three calibration methods an exterior orientation of the camera is estimated as a by-product of the calibration. This is necessary, because the exterior orientation is usually not known, but needed for the calculation of the ideal images. Usually some parameters of the exterior orientation are correlated with the parameters describing the position of the principal point. In (Gruen and Huang, 2001) this problem is addressed for test-field calibration in a chapter written by C. Fraser. According to his article, taking pictures with different roll angles leads to a decorrelation. The correlation between exterior and interior orientation is not restricted to test-field calibration but applies also to the goniometric calibration and the DOE calibration. Therefore a decorrelation by rolling the camera about the optical axis should be taken into account for these calibration methods as well.

\subsection{Test-field Calibration}

Test-field calibration is based on acquiring several images of a well known geometric pattern under different viewing angles. The position and viewing direction of each camera station is estimated. From the camera station and the known geometric pattern, an ideal image can be derived for each camera station by projecting the known pattern onto the focal plane using the colinearity equation. As a rule of thumb 8 to 12 images are needed for this calibration method. This type of calibration is also known as bundle-block-adjustment with self-calibration, details can be found in many standard books and papers, e.g. (Fraser, 1997), (Kraus, 2004).

Figure 1 shows the geometry of this calibration method with one example camera station.

\subsection{Goniometric Calibration}

Goniometric calibration uses a collimator producing a beam of parallel light simulating a light source (e.g. a spot) at infinity and a pan-tilt-table to rotate a mounted camera in a precise and well known manner within the collimated beam. The light spot is imaged by the optics of the camera onto the focal plane of the camera. The angular offsets between the viewing direction of the camera and the pan-tilt-table are estimated, these offset are equivalent to the exterior orientation of the camera. From the given angles of the pan-tilt-table the angle between the incoming beam and the viewing direction of the camera can be derived, which leads to the ideal image. Thus the viewing direction of a set of single pixels is determined (Schuster and Braunecker, 2000). For every angular setting of the pan-tilt-table an image has to be taken, which results in a huge number of images needed for this calibration method. To receive information about the whole sensor area, the pan-tilt-table must be operated accordingly. Preferably the illuminated pixels lie on a regular grid. In former times calibrations were based on measuring along the diagonals of the image, as stated for example in (Hallert, 1968). Overall this calibration method is very time consuming due to the large number

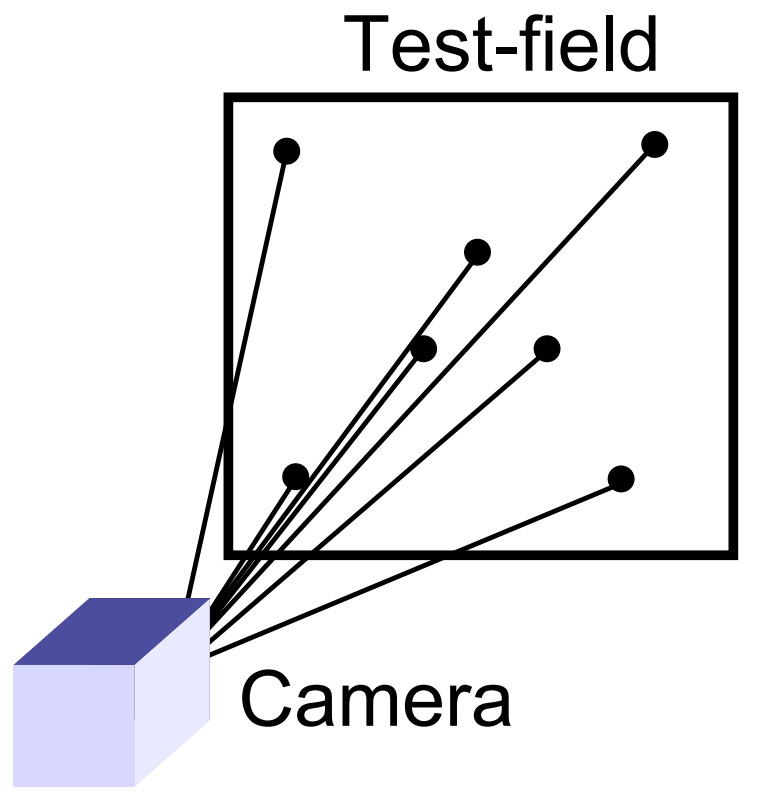

Figure 1: Schematic of the test-field calibration setup

of images needed, on the other hand it offers some interesting option, like monochromatic measurements and a wide range of calibratable focal lengths.

To compensate the aforementioned correlation between exterior and interior orientation it is sufficient taking at least two sets of measurements such that they differ by a rotation of about $90 \mathrm{deg}$ around the optical axis. The two sets should be aligned approximately to the rotation direction of the pan-tilt-table. In experiments using two sets of measurements instead one set, the correlation could be reduced from about 0.98 to about 0.5 .

Figure 2 shows the schematic experiment setup of the goniometric calibration. The collimator produces a beam of parallel light. The camera is rotated within the beam around two axis.

\section{Collimator}

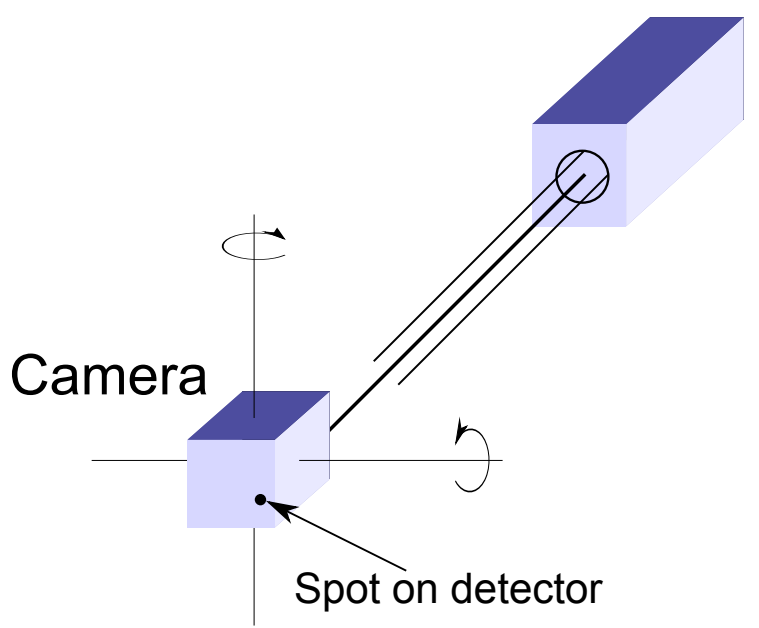

Figure 2: Schematic of the goniometric calibration setup 


\subsection{DOE Calibration}

DOE calibration uses a widened laser beam which passes through a diffractive optical element (DOE) producing a well known pattern of spots in the focal plane of the camera. An introduction to this calibration method can be found in (Krüger et al., 2005). The angle between the laser beam and the DOE as well as the viewing direction of the camera towards the DOE are estimated. The geometry of the DOE determines the spot pattern (Grießbach et al., 2010) from which an ideal image can be derived. This calibration methods only needs one single image.

Objectives with larger focal lengths are known to have little radial distortion, which makes it very difficult to determine the principal point due to the strong correlation between exterior and interior orientation. This correlation problem could be addressed probably in the same way as mentioned above by taking several pictures under different roll angles. This could not be experimentally tested yet.

\subsection{Calibration Method Characteristics Comparison}

In table 1 some characteristics of the investigated calibration methods are displayed. The table reveals some key advantages and disadvantages of each methods in comparison to the other methods.

\subsection{Distortion Model}

The distortion of a camera can be described by different means. For single-line detectors (e.g. CCD-line) it is quite usual to store the distortion description as a look-up-table or a function fitted along the explicitly measured pixel positions and their corresponding offsets. For matrix detector cameras, as investigated in this paper, it is usual to describe the distortion with a parametric model. In this paper one distortion model is used throughout all three calibration methods. It consists of 9 parameters proposed among others by D. C. Brown and selected by C. Fraser (Fraser, 1997). This model is used by the photogrammetric software Australis (see (Australis, 2001)). It describes radial-symmetric and radialasymmetric distortion as well as affinity and shear:

$$
\begin{aligned}
x_{\mathrm{corr}}:= & x+x \cdot d r+p 1 \cdot\left(r^{2}+2 \cdot x^{2}\right)+p 2 \cdot 2 \cdot x \cdot y \\
& +b 1 \cdot x+b 2 \cdot y \\
y_{\mathrm{corr}}:= & y+y \cdot d r+p 2 \cdot\left(r^{2}+2 \cdot y^{2}\right)+p 1 \cdot 2 \cdot x \cdot y
\end{aligned}
$$

Using following expressions:

$$
\begin{gathered}
x:=x_{\text {meas }}-x p \quad y:=y_{\text {meas }}-y p \\
r^{2}:=x^{2}+y^{2} \\
d r:=k 1 \cdot r^{1 \cdot 2}+k 2 \cdot r^{2 \cdot 2}+k 3 \cdot r^{3 \cdot 2}
\end{gathered}
$$

Where $x p, y p$ describes the principal point of the camera. The radial-symmetric distortion is described by $k 1, k 2, k 3$, the radialasymmetric distortion is described by $p 1, p 2$, affinity by $b 1$ and shear by $b 2$.

The set of distortion parameters resulting from a calibration describes the distortion of a camera. During the estimation of the distortion parameters the position of the measured geometric features is usually adjusted as well. The RMS of these geometric feature position adjustments (image coordinate residuals) can be used as an indicator for the quality of the calibration. The less the RMS is, the better the distortion parameters describe the observed distortion.

\subsection{Distortion Model Reduction}

The distortion of many objectives can be described with less than the 9 parameters included in the used distortion model. In order to prevent overfitting and to yield a compact model, it is a useful to reduce the set of distortion parameters, if possible. The selection can be driven by statistical tests or more empirically by considering the maximum contribution to the distortion description made by a certain distortion parameter. The influence of each distortion component increases towards the edges of the image. Therefore the maximum contribution is primarily given by the size of the sensor area. A parameter is indicated to be irrelevant, if its maximum contribution to the distortion is less than one pixel or if its contribution is far less than the contribution of other parameters.

For both methods it should be tested finally, if the resulting reduced model describes the distortion not much worse than the full model. A good measure for this is again the RMS of the image coordinate residuals, as introduced in the section above. The RMS of the reduced model is usually slightly greater than the RMS of the full model, but should be approximately the same, if the reduced model includes all relevant parameters.

All model reductions used in this paper are based on the empirically driven reduction.

\subsection{Distortion Parameter Estimation}

As mentioned above, the unknown parameters for exterior orientation, focal length and distortion are estimated from a comparison of an ideal image and a corresponding real image. To reduce the impact of measurement errors and to make use of the measurement redundancy, all investigated evaluation algorithms are based on least-square-adjustment.

The functional model is comprised by a difference of one expression for the ideal image and one expression for the distortioncorrected real image.

$0=$ ideal image feature - correct_distortion(real image feature)

Where the ideal image feature depends on the calibration method and the latter part is common among all three methods, as it describes the distortion model. As the distortion model operates independently in two different directions in the image, there are two equations needed for the distortion model (compare to 2.5) as well as this functional model. This generic approach allows other methods to be integrated easily into a further comparison. Only a mathematical description of the ideal images is needed.

\section{COMPARISON OF METHODS}

The following comparison is based on measurements with

- SMX-160M (SMX) with 2208 x 3000 pixels with $3.5 \mu \mathrm{m}$ pixel-size with objectives of following focal lengths:

- $4.8 \mathrm{~mm}(\mathrm{k} 1, \mathrm{k} 2, \mathrm{~b} 1)$

- Fairchild CAM 2051 (FC) with 2560 x 2160 pixels with $6.5 \mu \mathrm{m}$ pixel-size with objectives of following focal lengths:

- $18 \mathrm{~mm}(\mathrm{k} 1, \mathrm{k} 2)$

- $85 \mathrm{~mm}(\mathrm{k} 1, \mathrm{p} 1, \mathrm{~b} 1)$

- $300 \mathrm{~mm}(\mathrm{k} 1, \mathrm{p} 1, \mathrm{~b} 1)$ 


\begin{tabular}{|c|c|c|c|}
\hline Feature & Test field & Goniometric & DOE \\
\hline $\begin{array}{l}\text { Maximum FOV of the cam- } \\
\text { era }\end{array}$ & $360 \operatorname{deg} \times 180 \mathrm{deg}$ & $\begin{array}{l}\text { Restricted to the range of the } \\
\text { goniometer } 360 \mathrm{deg} \times 180 \\
\text { deg, depends on goniometer }\end{array}$ & $\begin{array}{l}<180 \text { deg } \times 180 \text { deg, depends } \\
\text { on the DOE }\end{array}$ \\
\hline $\begin{array}{l}\text { Maximum aperture diame- } \\
\text { ter of the camera }\end{array}$ & No hard restrictions & $\begin{array}{l}\text { Restricted by the diameter of } \\
\text { the collimated beam }\end{array}$ & $\begin{array}{l}\text { Restricted by the diameter of } \\
\text { the DOE }\end{array}$ \\
\hline $\begin{array}{l}\text { Maximum focal length, cal- } \\
\text { ibration in laboratory }\end{array}$ & $\begin{array}{l}\text { Approx. } 30 \mathrm{~mm} \text {, test-fields } \\
\text { for airborne images exist }\end{array}$ & $\begin{array}{l}\text { Up to some } 100 \mathrm{~mm} \text {, depends } \\
\text { on focal length of collimator } \\
\text { and precision of goniometer }\end{array}$ & Depends on DOE \\
\hline Spectral range & $\begin{array}{l}\text { Usually white, restriction by } \\
\text { filtering the illumination }\end{array}$ & $\begin{array}{l}\text { White, mixed, monochro- } \\
\text { matic }\end{array}$ & Monochromatic (Laser) \\
\hline Laboratory Setup & $\begin{array}{l}\text { Test field, (reference distance } \\
\text { measure), illumination }\end{array}$ & $\begin{array}{l}\text { Goniometer, Collimator, } \\
\text { Light source, (Filter) }\end{array}$ & DOE, Laser, Optics \\
\hline Costs for Laboratory Setup & Low & High & High \\
\hline Geometric Features & $\begin{array}{l}\text { Usually Circles, Squares or } \\
\text { Lines; sometimes coded for } \\
\text { automatic identification }\end{array}$ & Light Spot & Light Spots \\
\hline $\begin{array}{l}\text { Geometric Features per Im- } \\
\text { age }\end{array}$ & $20-500+$ & 1 & $200-3000+$ \\
\hline Images per Calibration & $\begin{array}{l}8-20, \text { one-shot calibration } \\
\text { exists, but is less reliable }\end{array}$ & $>100$ & 1 \\
\hline $\begin{array}{l}\text { Costs per Calibration (Im- } \\
\text { age acquisition) }\end{array}$ & $\begin{array}{l}\text { Medium: } 8-12 \text { images, with } \\
\text { sufficient roll-diversity }\end{array}$ & High: Many images & Low: One single image \\
\hline $\begin{array}{l}\text { Costs per Image (Image ac- } \\
\text { quisition) }\end{array}$ & $\begin{array}{l}\text { Low: No precise alignment } \\
\text { necessary }\end{array}$ & Low: Move goniometer & $\begin{array}{l}\text { Low: No precise alignment } \\
\text { necessary }\end{array}$ \\
\hline $\begin{array}{l}\text { Costs per Geometric Fea- } \\
\text { ture (Image acquisition) }\end{array}$ & $\begin{array}{l}\text { Low: many marker in one im- } \\
\text { age }\end{array}$ & $\begin{array}{l}\text { High: only one feature per im- } \\
\text { age }\end{array}$ & $\begin{array}{l}\text { Low: all observable features } \\
\text { in one image }\end{array}$ \\
\hline $\begin{array}{l}\text { Automated image acquisi- } \\
\text { tion }\end{array}$ & $\begin{array}{l}\text { Fairly possible: move camera } \\
\text { and/or test field }\end{array}$ & $\begin{array}{l}\text { Possible: move goniometer, } \\
\text { take image }\end{array}$ & $\begin{array}{l}\text { Possible: take image (take } \\
\text { several pictures with different } \\
\text { integration times) }\end{array}$ \\
\hline Image Analysis & $\begin{array}{l}\text { Find, identify and label } \\
\text { marker }\end{array}$ & Find spot & $\begin{array}{l}\text { Find spots, identify neigh- } \\
\text { bourhood relationships }\end{array}$ \\
\hline Automated Image Analysis & Possible: coded targets & $\begin{array}{l}\text { Possible: Prediction of spot } \\
\text { position from goniometric } \\
\text { measurement }\end{array}$ & $\begin{array}{l}\text { Possible: Iterative spot identi- } \\
\text { fication }\end{array}$ \\
\hline $\begin{array}{l}\text { Costs per Calibration (Im- } \\
\text { age Analysis) }\end{array}$ & Medium & Medium: Many images & Medium \\
\hline $\begin{array}{l}\text { Costs per Image (Image } \\
\text { Analysis) }\end{array}$ & Medium & Low & Medium \\
\hline $\begin{array}{l}\text { Costs per Geometric Fea- } \\
\text { ture (Image Analysis) }\end{array}$ & Medium: find, identify, label & Low: Find & $\begin{array}{l}\text { Medium: Find and identify } \\
\text { neighbourhood relationship }\end{array}$ \\
\hline
\end{tabular}

Table 1: Characteristics of calibration methods 
In parenthesis the parameters of the reduced model are indicated. Focal length and principal point are always included.

With every calibration the RMS of image coordinate residuals is calculated in fractions of the pixel size. This is a comparable indicator for the quality of the calibration. The results are listed in table 2. The values given result from the application of a full / a reduced / no distortion model. If no distortion correction is applied, only the focal length and the principal point are estimated.

\begin{tabular}{|c||r|r|}
\hline Camera / Objective & Test-field & Goniometric \\
\hline \hline SMX / $4.8 \mathrm{~mm}$ & $0.62 / 0.62 / 11.33$ & $0.72 / 0.91 / 49.08$ \\
\hline $\mathrm{FC} / 18 \mathrm{~mm}$ & $0.66 / 0.67 / 6.07$ & $0.86 / 0.92 / 3.07$ \\
\hline FC / $85 \mathrm{~mm}$ & & $0.27 / 0.29 / 0.57$ \\
\hline FC / $300 \mathrm{~mm}$ & & $0.14 / 0.15 / 0.52$ \\
\hline
\end{tabular}

Table 2: RMS of image coordinate residuals from calibration in pixel. Full / reduced / no distortion correction.

As a geometric calibration is used to compensate the distortion, the quality of a calibration can also be measured by the ability of undistorting an independent test image, that was not part of the calibration. This means for each calibration method one set of images must be taken for calibration and another independent set of images must be taken for verification. The verification is calculated using the same calibration algorithms, except that the distortion parameters are not estimated, but taken as fixed input from the previous calibration. The RMS of the image coordinate residuals from the independent test image is used as a measure of quality and can be compared from one method to another.

This comparison can be generalized to a cross-method comparison. It is basically achieved in the same way as described for the intra-method verification. The difference is, that the distortion parameters are taken from a different calibration method. Thus an independent test image is processed with a set of distortion parameters gained by a another calibration method. This comparison is displayed only for the FC / $18 \mathrm{~mm}$ camera in table 3 . The main diagonal of this matrix contains the results of the intramethod verification.

\begin{tabular}{|c||r|r|r|}
\hline Taken from $\backslash$ Verified in & Test-field & Goniometric & DOE \\
\hline \hline Test-field & 0.48 & 1.30 & 0.12 \\
\hline Goniometric & 0.65 & 0.62 & 1.64 \\
\hline DOE & 0.55 & 1.11 & 0.25 \\
\hline
\end{tabular}

Table 3: RMS of image coordinate residuals from cross-method verification in pixel. Reduced distortion models applied.

\section{CONCLUSION}

All three investigated calibration methods (test-field calibration, goniometric calibration and DOE calibration) provide sets of distortion parameters with high accuracy. The calibration method can be choosen almost freely due to the requirements of a certain calibration task. It is a known fact, that the distortion does not depend only on the position in the image. The distance between the object and the camera has to be taken into account as well. Table 3 indicates, that test-field calibration and DOE calibration are best choices for close-range photogrammetry in contrast to the collimator-goniometric calibration, which is the best choice for cameras looking to infinity. At this point further investigation can be driven towards goniometric calibration with a close-range target instead of a collimator. Alternatively a collimator with well-defined, non-parallel light could be used for close-range calibration.

\section{ACKNOWLEDGEMENTS}

This work has been supported by Humboldt-Universität zu Berlin and the German Aerospace Center (DLR).

\section{REFERENCES}

Australis, 2001. Image coordinate correction function in australis.

Clarke, T. A. and Fryer, J. G., 1998. The Development of Camera Calibration Methods and Models. The Photogrammetric Record 16(91), pp. 51-66.

Fraser, C. S., 1997. Digital camera self-calibration. In: ISPRS Journal of Photogrammetry and Remote Sensing, Vol. 52(4), pp. 149-159.

Grießbach, D., Bauer, M., Scheele, M., Hermerschmidt, A. and Krüger, S., 2010. Geometric stereo camera calibration with diffractive optical elements. In: Proceedings of the joint EuroSDR/ISPRS 'International Calibration and Orientation Workshop EuroCOW 2010'.

Gruen, A. and Huang, T. S., 2001. Calibration and Orientation of Cameras in Computer Vision. Springer Series in Information Sciences, Vol. 34, Springer.

Hallert, B., 1968. Notes on calibration of cameras and photographs in photogrammetry. Photogrammetria 23, pp. 163-178.

Kraus, K., 2004. Photogrammie: Geometrische Informationen aus Photographien und Laserscanneraufnahmen. 7th edn, Walter de Gruyter.

Krüger, S., Scheele, M. and Schuster, R., 2005. New calibration scheme for panoramic line scanners. In: INTERNATIONAL ARCHIVES OF PHOTOGRAMMETRY, REMOTE SENSING AND SPATIAL INFORMATION SCIENCES, Vol. XXXVI-5/W8.

Luber, A. and Reulke, R., 2010. A unified calibration approach for generic cameras. In: International Archives of Photogrammetry, Remote Sensing and Spatial Information Sciences, Vol. XXXVIII, Part 5, pp. 399-404. Commission V Symposium, Newcastle upon Tyne, UK. 2010.

Schuster, R. and Braunecker, B., 2000. Calibration of the lh systems ads40 airborne digital sensor. In: International Archives of Photogrammetry and Remote Sensing. Vol. XXXIII, Part B1. Amsterdam, 2000, Vol. Vol. XXXIIInumber Part B1, pp. 288- 